\title{
Crowdsourcing for individual needs - the case of routing and navigation for mobility-impaired persons
}

\author{
Alexander Zipf ${ }^{\star}$, Amin Mobasheri, Adam Rousell and \\ Stefan Hahmann \\ GIScience research group, Institute of Geography, Heidelberg University, \\ Heidelberg, Germany \\ *zipf@uni-heidelberg.de
}

\begin{abstract}
Routing and navigation web services are becoming widely used, and make use of both commercial and VGI datasets. It is now becoming widely acknowledged that a 'one fits all' method of generating and presenting routes is not applicable. In particular, the accessibility of places for the mobility impaired has become a key focus with several services addressing topics such as how accessible locations of interest are and how to best generate routes for people who need to consider additional factors. Though datasources such as OpenStreetMap (OSM) are well suited for such topics, several issues including the quality of the underlying data remain. Through the use of quality assessment tools it is possible to identify areas with inadequate data completeness with regards to the information needed for the mobility impaired and thus encourage the enrichment of these areas through specialised tagging applications. Such data can then be used in routing and navigation services which focus on ensuring that routes being generated and presented fit the personal requirements of the traveller.
\end{abstract}

\section{How to cite this book chapter:}

Zipf, A, Mobasheri, A, Rousell, A and Hahmann, S. 2016. Crowdsourcing for individual needs - the case of routing and navigation for mobility-impaired persons. In: Capineri, C, Haklay, M, Huang, H, Antoniou, V, Kettunen, J, Ostermann, F and Purves, R. (eds.) European Handbook of Crowdsourced Geographic Information, Pp. 325-337. London: Ubiquity Press. DOI: http://dx.doi.org/10.5334/bax.x. License: CC-BY 4.0. 


\section{Introduction}

Routing and navigation services for vehicles and people are based on geospatial data. Due to the availability of GPS sensors within handheld devices, systems have been able to localise themselves precisely on the road. These services increasingly make use of public transport data and integrate near real-time traffic data. Also, through the development of new algorithms that employ hierarchical methods, routing has become faster, especially for calculating long distance routes. Nowadays, routing services are able to provide routes based on several criteria such as distance, road type, and traffic to an acceptable degree of accuracy. In addition, further research prototypes have identified a number of extra criteria. With the arrival of crowdsourcing and VGI (in particular the OpenStreetMap project), a new generation of route planning services using such services has emerged. As an example, OpenRouteService.org (Neis \& Zipf, 2008) used OSM as data source for deriving optimal routes between two locations for different modes of transport including car, several types of bikes, and pedestrians. The potential of crowdsourced geographic information for routing and navigation can be highlighted by the fact that the OSM based OpenRouteService ${ }^{1}$ was able to provide pedestrian and bicycle routing across several countries even before Google offered these features. This is because of the different way crowdsourced information like OSM is being collected. Volunteers do this on the ground (commonly on foot or bicycle) leading to a higher representation of this particular kind of data than offered by commercial providers before. This particular richness of VGI also offers new possibilities for even more specific information needs and specialised applications.

Besides the support of these mainstream route planning needs, routing services that serve people with special requirements (such as wheelchair routing) are being designed and developed. However, in order to provide the appropriate data required for such services that meet the specific information requirements of the respective users, new sources of information are required. For instance, in addition to road features, a wheelchair routing service would need to consider sidewalk data, such as curbs, surface type and incline information, to name a few. Müller et al. (2010) therefore suggested several extensions to the OSM tagging schema including new tags, as well as identifying a selection of relevant already existing ones. This supported OSM mappers in the collection of such information. These tags have been added to the OSM schema and are described in the OSM wiki². Such detailed information is however, still not generally available or complete in the OpenStreetMap dataset. Therefore, special crowdsourcing tools and services are necessary in order to better support the collection of geospatial data relevant for routing and navigation of people with limited mobility.

\footnotetext{
${ }^{1}$ http://www.openrouteservice.org

${ }^{2}$ http://wiki.openstreetmap.org/wiki/Wheelchair_routing
} 
To address the incompleteness of data it is necessary to develop and pilottest methods and tools for collectively gathering and sharing spatial information for improving accessibility. The power of online maps and mobile devices can help foster an awareness of barriers for individuals with limited mobility and encourage the removal of such barriers. As this is highly relevant for an increasing proportion of our society, the European Commission has decided to fund projects in this domain. One example is the CAP4Access ${ }^{3}$ project. The agenda of research and development in this field includes the design and implementation of tools and methods for (a) quality assessment, i.e. checking the completeness of OSM data with regard to required information; b) tagging, i.e. describing and discussing locations and routes within the built environment according to their accessibility; (c) route planning and navigation; (d) raising awareness and preparing effective measures at local level for eliminating barriers. Target groups include people requiring enhanced accessibility, grassroots initiatives supporting people with disabilities, policy-makers, planners and service providers with responsibility for the built environment, and the general public.

\section{Required and existing services}

\section{Quality assessment and enrichment of crowd-sourced data}

Ensuring the quality of crowdsourced data is of particular importance in order to ensure that the results of routing and navigation services offered are accurate and ultimately useful. Generally, routing and navigation services for people with limited mobility benefit from information regarding new obstacles provided by involved communities. However, crowdsourced data has significant differences from traditional geospatial data which are often created by specifically dedicated organizations and experts, and are generated according to standardised structures and languages. Especially in the case of routing tools, completeness of data and spatial accuracy is of great concern to ensure proper routing. The development of a data quality assessment component is therefore a crucial objective.

There are a number of different geo-data quality elements that one might need to check before using any kind of datasets in their project. These elements include positional accuracy, attribute accuracy, completeness, logical consistency and temporal accuracy (van Oort, 2006). A discussion on all data quality elements are out of the scope of this chapter. However, as an example we provide information on one of the important data quality elements for routing and navigation services - completeness. Completeness is defined as errors of omission (measure of the absence of data), and errors of commission

\footnotetext{
${ }^{3}$ http://myaccessible.eu/
} 
(measure of the presence of extra data) (van Oort, 2006). The completeness of a dataset can be suitable for a specific task but not for another. So, when completeness has to be measured, the concept of fitness for use comes in mind.

In order to check the completeness of a dataset three tests could be performed:

- missing object/line: here we find missing information at object level, whether a line or polygon is missing in the dataset. This should be done by using a reference dataset if possible.

- missing attribute: for those objects (point/line/polygon) that are available we need to know which attributes are missing (based on a list of attributes that are important and used by the routing and navigation system). Missing attributes are counted as inconsistencies and reported. This check can be performed by automated means of intrinsic data check, which means that only the dataset itself is investigated (Barron et al. 2013).

- missing value: From those existing attributes, some may be incomplete in terms of missing values. Here we check those attributes/fields that lack value

There are several tools that could be used for checking the completeness of an OpenStreetMap dataset. For example, OSMatrix ${ }^{4}$ is a tool for visualising mapping progress/quality on various metrics (Roick et al. 2011, 2012). By using OSMatrix, one can calculate the number of certain object features in OSM (e.g. sidewalks) at various timestamps. For example, Figure 1 shows a snapshot of visual and statistical information regarding the total number of sidewalk information (tags in OpenStreetMap related to sidewalk information) for a selected region in Heidelberg, in Jan 2016. The sample area is divided into hexagonal cells with the size of $1 \mathrm{~km}$. Each cell shows a value representing the aggregation of the total number of tags in OSM related to sidewalk attributes in that hexagon. OSMatrix could also be used in order to derive statistical and visual information regarding the completeness of OSM data at the attribute level (e.g. sidewalk width, incline, etc.).

As another example of tools, OSM Quality Assurance Editor ${ }^{5}$ can be used to understand the completeness of certain object features in OSM data using an object-based approach. Figure 2 shows a region in Heidelberg (Germany) where sidewalk information is missing (the road features contain no information regarding the presence or absence of sidewalks attached to the road). This information is given per object, meaning that one could select an object and view its properties as opposed to the provision of an aggregated region-wide value. This is a large benefit in comparison to OSMatrix is in that tool the statistical information is aggregated and provided for each cell, while information regarding route objects inside the cells cannot be realised.

\footnotetext{
${ }^{4}$ http://alborz.geog.uni-heidelberg.de/osmatrix/

http://editor.osmsurround.org/
} 


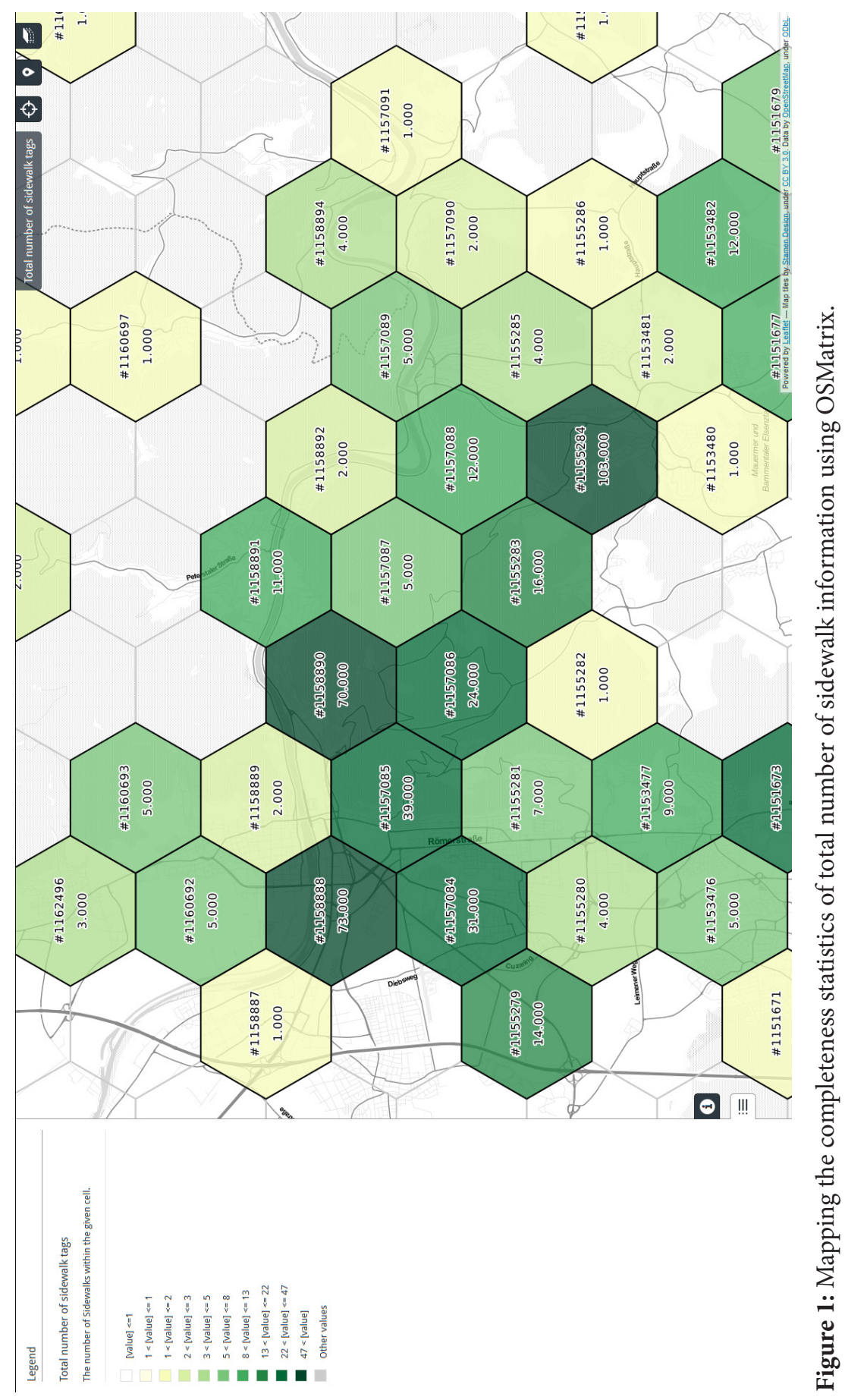




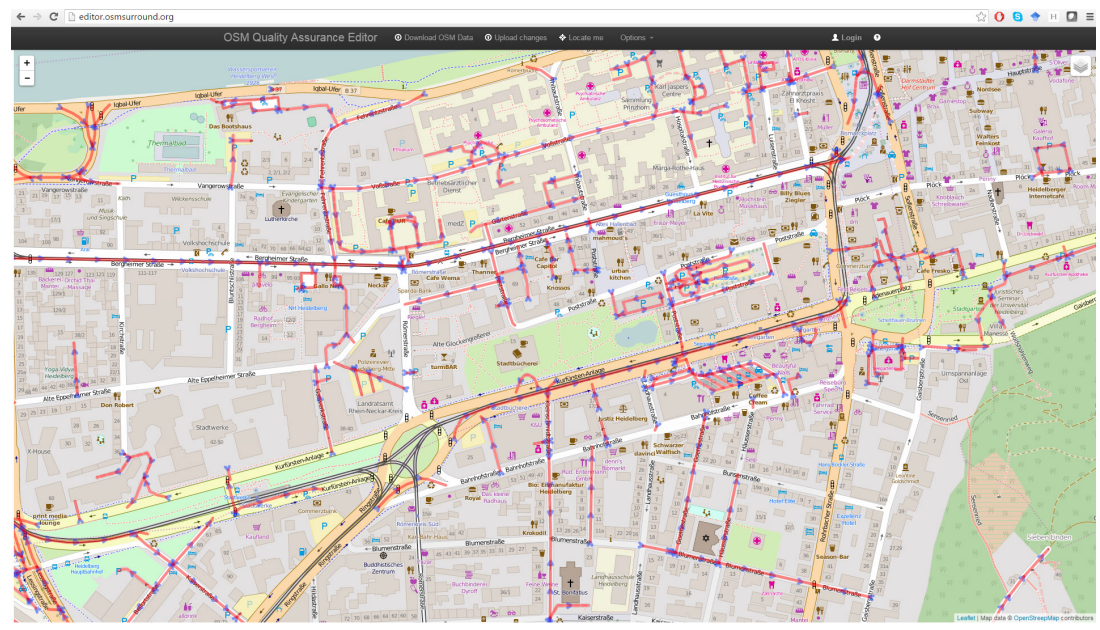

Figure 2: An example of selected road features that have no information regarding existence of sidewalk.

In order to provide an effective routing and navigation service for people with limited mobility, it is crucial to have data regarding sidewalk features and their attributes such as surface texture, width, incline, etc. The quality assessment tools can aid in the understanding of the level of incompleteness of such information within OpenStreetMap data, as well as identifying the places where such information is missing. In order to better inform the user about the potentially non-perfect data for wheelchair routing, Neis (2014) developed an initial prototype of a reliability index that attempts to measure some aspects regarding the information quality of the selected route.

With regards to data availability, Figure 2 shows that most road and streets in the selected region in Heidelberg are missing sidewalk information. Therefore, in order to collect and enrich OSM with sidewalk information a tagging system is suggested. Tagging systems are developed to support the collection of user-generated data. In the case of the restricted mobility topic, this data focusses on the accessibility of places, points of interest and roads. The collective tagging approach has already been applied within the accessibility theme through the Wheelmap ${ }^{6}$ platform. Wheelmap is a map for wheelchair-accessible places. Locations are rated and portrayed according to a traffic light system based on their accessibility status (e.g. accessibility of restaurants for people on wheelchairs). One of the disadvantages of Wheelmap with regards to collecting accessibility information for improving routing and navigation of people with

\footnotetext{
${ }^{6}$ http://wheelmap.org/
} 
restricted mobility is that it is not capable of collecting accessibility information for linear objects such as sidewalks.

Since sidewalk information is crucial to routing services that need to provide the most accessible route from one location to another, other tagging systems should be used that provide the capability of enriching sidewalk information such as (for example) availability of a sidewalk, sidewalk width, incline and surface texture. For this purpose, any OSM editor that could be used for editing line features (e.g. Vespucci ${ }^{7}$ OSM editor, JOSM, etc.) can be employed.

\section{Routing}

A routing service tailored to special needs is a core service to improve the mobility of people with various types of disabilities (Neis and Zielstra 2014). A routing system mostly consists of two core components: a graph network representing the underlying street datasets, and a routing engine that uses this graph to generate feasible routes.

There are a number of available routing services described on the OSM Wiki pages $^{8}$ that make use of OSM data. However, of the 13 route services documented there only three currently provide the functionality to be extended for to address specialised requirements, such as wheelchair routing. These three are OpenRouteService, Routino ${ }^{9}$ and OpenTripPlanner ${ }^{10}$. OpenRouteService. org (ORS; Neis \& Zipf 2008) is built according to open standards from the Open Geospatial Consortium (OGC) meaning that it can easily be integrated in other applications or regional web sites. In order to use the crowdsourced data from OSM for wheelchair routing, applications such as ORS have been (and will need to further be) extended so that they can be used by persons with limited mobility with various profiles and parameters. Figure 3 shows the result of a route plan where a difference between the pedestrian profile and the wheelchair profile occurs due to a pedestrian bridge that is only accessible via steps which are not feasible for wheelchair users. A first prototype for wheelchair routing based on OpenRouteService was developed earlier by Müller et al. $(2010)^{11}$.

The main challenge to serve special route planning requirements is the need for very specific data (e.g. sidewalk data), which is not available for large areas, e.g. country-wide. This especially includes data about sidewalks (e.g. width, surface, smoothness, incline and existence of sidewalks) and also the position and height of sloped/dropped curbs. Such data is not usually published in authoritative products due to their scope, even though the data is often available

\footnotetext{
${ }^{7} \mathrm{http} / / /$ wiki.openstreetmap.org/wiki/Vespucci

${ }^{8} \mathrm{http} / / /$ wiki.openstreetmap.org/wiki/Routing/online_routers

${ }^{9} \mathrm{http} / / /$ www.routino.org/

${ }^{10} \mathrm{http} / / /$ www.opentripplanner.org/

${ }^{11}$ http://rollstuhlrouting.de
} 


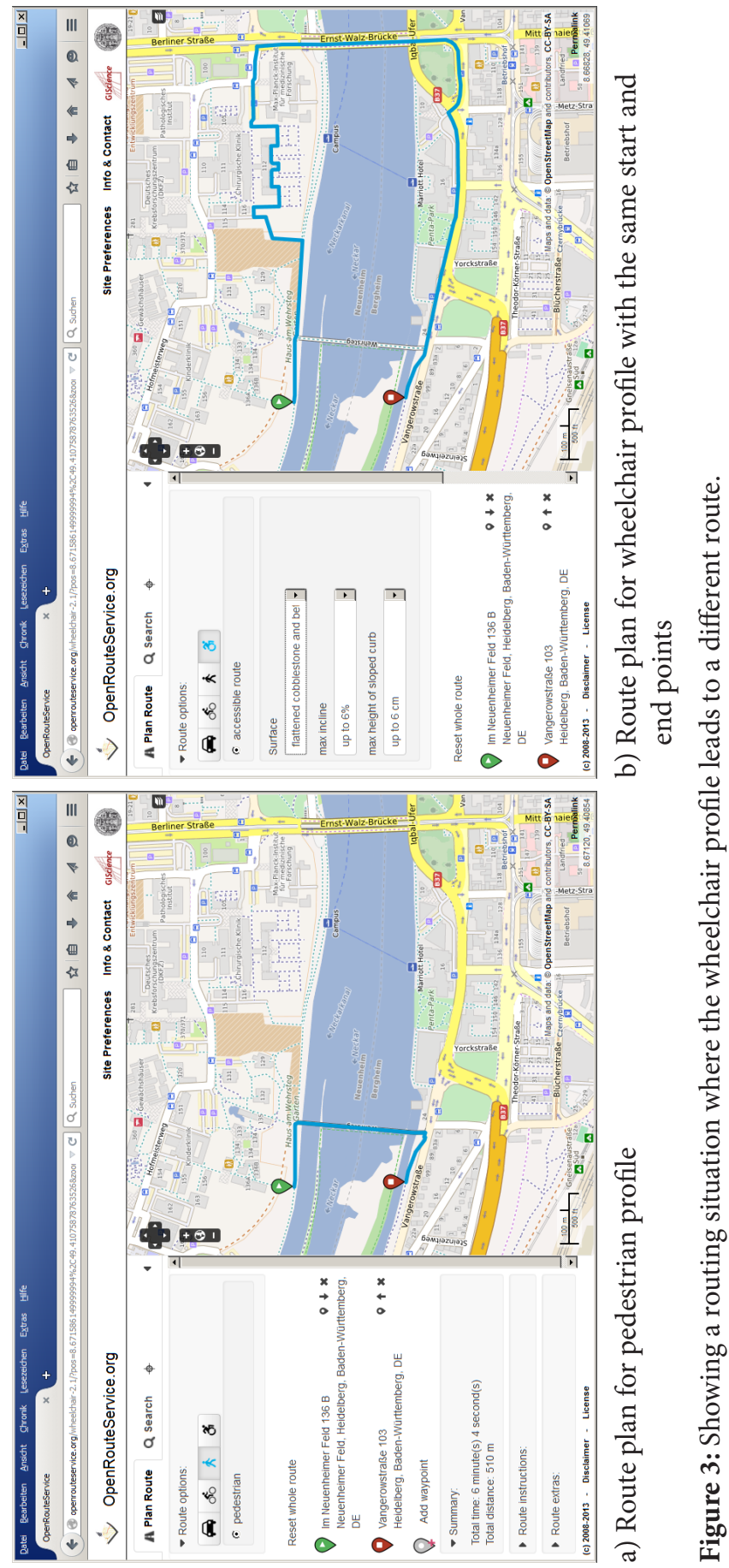


within the organisation. Even if these datasets were made available however, the heterogeneity between data structures would make integration problematic. Considering this situation, VGI becomes an important data source as local knowledge from the users can be harnessed. Care needs to be taken however to provide feedback (in the form of changes to existing services) to the volunteers to ensure that their motivation is maintained. In terms of the sidewalks themselves, the OSM community has decided that within their dataset, apart from defined exceptions, sidewalks should not be represented by separate features, but instead through attributes held by their associated roads.

Another challenge relating to routing (in particular for pedestrians) is that existing routing algorithms can only process data that is provided in a line format. Within OSM, many traversable surfaces (such as city squares and parks) are represented as polygon areas which most routing algorithms simply do not know how to handle. Several methods could be implemented to generate linear representations of these spaces such as using the polygon outline (figure 4a), or generating internal linear structures through grids (figure 4c), skeletons (figure 4d) or lines-of-sight.

\section{Navigation}

Although routing systems provide a valuable service with regards to getting around unknown environments, the determination of a route is only one step in an overall wayfinding process. As well as being able to calculate a route through space that is suitable for an individual's preferences, this information needs to be conveyed in a format that allows them to successfully traverse the intended route. Many navigation systems exist which attempt to provide direction instructions to travellers, often in the form of distance and street names. It is well documented that when describing directions (particularly for pedestrians) landmarks form an essential component of the descriptive (Duckham et al. 2010, Winter et al. 2005). Proper selection of landmarks in navigation of people with restricted mobility is challenging since it requires special considerations compared to normal pedestrians. For instance, the fact that people on wheelchair have less visibility than those who are standing is an issue that must be considered.

A number of methods have been identified that extract landmarks that can be used for navigational instructions, some of which make use of VGI datasets (such as Dräger and Kroller 2012). Key considerations when producing such methods include the scalability, performance in terms of determination speed, and the suitability of the actual landmarks identified.

With regards to navigation services for mobility impaired users, it is important to take into account the individual requirements of each user and then feed these through to the underlying route generation system. The system should also allow users to report any obstacles that they encounter which were not included in the route generation due to the incompleteness of the underlying 


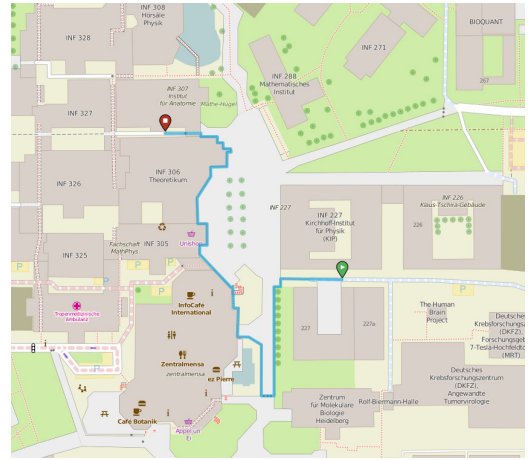

a) Planned route using the outline of an open space area

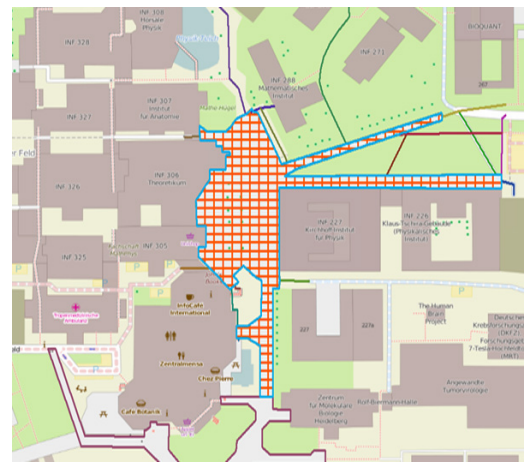

c) Grid (orange) based approach as a subgraph of the polygon (light blue)

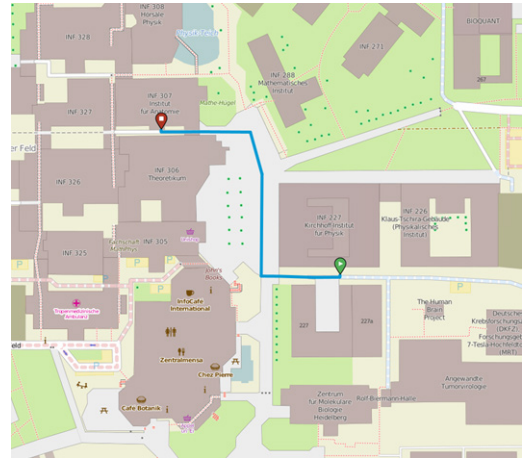

b) Desired route going through the open space area

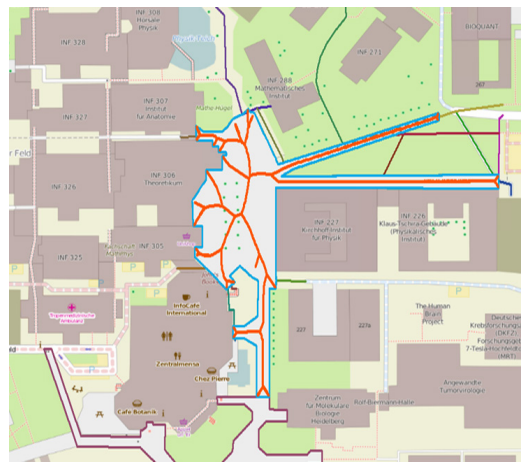

d) Skeleton (orange) based approach as a subgraph of the polygon (light blue)

Figure 4: Different routing approaches through polygons (grid, skeleton, line of sight).

dataset. This in turn not only empowers the user in terms of them making a difference to the system, but it also increases the overall completeness of the source dataset.

One of the main challenges posed currently is how VGI and social media can be used to identify suitable landmarks that can be used in navigational instructions. Not only does this require the fusion of freely available data from various sources (i.e. OSM, FourSquare and Twitter), it also generates technical problems such as providing a fast lookup service for possible candidates from a population. Overall, what we want is a system that can quickly provide an instruction for any route similar to "just after $x x x$, turn left" where $x x x$ is the most salient object in relation to the turning point. 


\section{Discussion and Conclusion}

Routing and navigation services are becoming more personalized and near real-time, by recommending routes that take into account various preferences and up-to-date information. This has long been argued for in the field of adaptive and personalized Location Based Services (LBS) (Malaka and Zipf 2000, Zipf 2002). In contrast to former times, such developments can now be realised much easier due to the wealth of rich data that is made available through VGI and the Social Web.

In this chapter, the potentials of using crowdsourced geo-information for routing and navigation with a focus on people with restricted mobility were presented. Although the completeness of OpenStreetMap data with regard to specific information for those user groups such as relevant barriers or details on sidewalks still is low, there are developments under way to improve the situation both in an automated way as well as by harnessing the power of the crowd, so we can expect more and more areas where enough information is available. We explored the OSMatrix Web-Service and presented how such a portal visualizing intrinsic quality indicators can be used in order to help understand the data and attribute availability for certain features (e.g. sidewalks). Furthermore, we see the need for further research in the area of (semi-) automated data integration from different user generated data sources in order to further enrich the routing graph with relevant objects and attributes.

As an example related to both pedestrian and wheelchair navigation, we discussed the problems on deriving routing graphs dealing with with open spaces (e.g. city squares and parks). For this possible ideas for generating routing graph were explained. These are further investigated and evaluated with respect to efficiency and quality in our current research. It was discussed that while routing services provide valuable information, they are only the first step in the overall wayfinding process. A navigation service is an additional component that receives routing information as input, processes, translates and communicates the information in a format that allows the individuals to traverse the relevant route. For this, the derivation and selection of landmarks from OSM and other crowdsourcing platforms is an interesting research topic. Regarding navigation services for mobility impaired users, it was concluded that individual requirements of each user need to be collected and considered in the process of route generation. Extraction of relevant information from both the users' context as well as the environment from VGI and crowdsourced data could lead to a richer real-time routing and navigation service that could benefit from the availability of temporally detailed road network data with conditions such as speed or other detailed semantic attributes.

\section{Acknowledgements}

The research leading to these results has received funding from the European Community's Seventh Framework Programme (FP7/2007-2013) under grant 
agreement $n^{\circ} 612096$ (CAP4Access). The work has been partly supported by funding from the Klaus-Tschira-Foundation (KTS), Heidelberg.

\section{References}

Barron, C., Neis, P., \& Zipf, A. 2013. A Comprehensive Framework for Intrinsic OpenStreetMap Quality Analysis. Transactions in GIS. DOI: http://dx.doi. org/10.1111/tgis.12073

Dräger, M., \& Koller, A. 2012. Generation of landmark-based navigation instructions from open-source data. In: Proceedings of the 13th Conference of the European Chapter of the Association for Computational Linguistics. Avignon, France: Association for Computational Linguistics, pp. 757-766.

Duckham, M., Winter, S., \& Robinson, M. 2010. Including landmarks in routing instructions. Journal of Location Based Services, 4(1): 28-52.

Grossner, K., \& Glennon, A. 2007. Volunteered geographic information: Level III of a digital earth system. In: Proceedings of the Position paper presented at the Workshop on Volunteered Geographic Information, Santa Barbara, CA, USA, pp. 13-14.

de Longueville, B., Ostlander, N., \& Keskitalo, C. 2009. Addressing vagueness in volunteered geographic information (VGI) - A case study. International Journal of Spatial Data Infrastructures Research. Special Issue GSDI-11.

Malaka, R., \& Zipf, A. 2000. DEEP MAP - Challenging IT research in the framework of a tourist information system. In: Fesenmaier, D., Klein, S., \& Buhalis, D. (Eds.) Information and Communication Technologies in Tourism 2000. Proceedings of ENTER 2000, Barcelona. Spain. Springer Computer Science, Wien, New York, pp. 15-27.

Müller, A., Neis, P., \& Zipf, A. 2010. Ein Routenplaner für Rollstuhlfahrer auf der Basis von OpenStreetMap-Daten. Konzeption, Realisierung und Perspektiven. AGIT 2010. In: Proceedings of Symposium für Angewandte Geoinformatik. Salzburg. Austria.

Neis, P. 2014. Measuring the Reliability of Wheelchair User Route Planning based on Volunteered Geographic Information. Transactions in GIS.

Neis, P., \& Zipf, A. 2008. OpenRouteService.org is three times "Open": Combining OpenSource, OpenLS and OpenStreetMaps. GIS Research UK (GISRUK 08). Manchester, UK.

Neis, P., \& Zielstra, D. 2014. Generation of a tailored routing network for disabled people based on collaboratively collected geodata. Applied Geography, 47: 70-77.

Roick, O., Hagenauer, J., \& Zipf, A. 2011. OSMatrix - Grid based analysis and visualization of OpenStreetMap. SOTM-EU 2011. State of the Map EU. Scientific Track. Wien. 
Roick, O., Loos, L., \& Zipf, A. 2012. Visualizing spatio-temporal quality metrics of Volunteered Geographic Information - A case study for OpenStreetMap. Geoinformatik 2012. Mobilität und Umwelt. Braunschweig. Germany.

van Oort, P. V. 2006. Spatial Data Quality: from Description to Application, $P h D$ dissertation, Wageningen Universiteit, Wageningen, The Netherlands.

Winter, S., Raubal, M., \& Nothegger, C. 2005. Focalizing Measures of Salience for Wayfinding. In: Meng, L., Reichenbacher, T., \& Zipf, A., (Eds.) Mapbased Mobile Services. Springer Berlin Heidelberg, pp. 125-139.

Zipf, A. 2002. User-Adaptive Maps for Location-Based Services (LBS) for Tourism In: Woeber, K., Frew, A., \& Hitz, M. (Eds.) Proc. of the 9th Int. Conf. for Information and Communication Technologies in Tourism, ENTER 2002. Innsbruck, Austria. Springer Computer Science. Heidelberg, Berlin. 